\title{
Effects of reward schedules on the acquisition of conditioned reward value
}

\author{
KATHRYN A. KENDALL and JUM C. NUNNALLY, Vanderbilt \\ University, Nashville, Tenn.
}

Forty college Ss learned to discriminate among four nonsense syllables, each associated with a different reward contingency: $100 \%$ reward, $50 \%$ reward, no reward, and loss of reward. Subsequently, measures were made of the "pleasantness" and reward expectancy that these syllables had acquired. The results offer considerable support for three major conclusions: as compared with a neutral object associated with reward $100 \%$ of the time, an object associated with reward $50 \%$ of the time (a) is seen as somewhat less pleasant, but still pleasant in an absolute sense, (b) has a lower expectancy as a cue to future reward, but elicits a positive expectancy in an absolute sense, and $(c)$ is seen as relatively mysterious or difficult to understand. These results are discussed as being more readily explained in terms of classical conditioning rather than in terms of frustration or cognitive dissonance theories.

Previous studies have demonstrated that neutral objects (such as nonsense syllables) may acquire rewarding and punishing properties through association with the gain or loss of rewards. Furthermore, these objects are then capable of eliciting numerous responses (e.g., attentional, affective, cognitive) which reflect the attributes conditioned to them. This effect has been termed conditioned reward value, and prior investigations have examined some of the parameters of the conditioning and of the resultant behaviors (e.g., Nunnally, Duchnowski, \& Parker, 1965; Nunnally, Stevens, \& Hall, 1965; Parker \& Nunnally, 1966; Nunnally \& Faw, 1968).

The present study is concerned with the effects that a partial schedule of reward introduces to conditioned reward value, in comparison to the effects produced by a continuous schedule of reward. Four nonsense syllables were employed in a discrimination task, each with a different reward contingency: $100 \%$ reward, $50 \%$ reward, no reward (neutral), and loss of reward. Subsequently, measures were made of the "pleasantness" that these syllables had acquired as a result of the associations. In addition, measures were made of S's expectancy that further rewards would be associated with the different syllables.

Three possible outcomes of this experiment have implications for three theoretical positions regarding the effect of a partial schedule of reward on conditioned reward value. The most explicit prediction follows from the literature on classical conditioning. The prediction is that the partially rewarded syllable will acquire pleasantness in an absolute sense (i.e., it will be more pleasant than the neutral syllable) but will be distinctly less pleasant than the continuously rewarded syllable.

If the partially rewarded syllable proved to be unpleasant, this finding could not be rationalized by the literature on classical conditioning. It could, however, easily be rationalized by theories of frustration relating to partial schedules (e.g., Amsel, 1962; Longstreth, 1966). The frustration (an aversive emotion) occurring on non-rewarded trials could, supposedly, "rub off" on the syllable associated with the partial schedule.

If the partially rewarded syllable proved to be even more pleasant than the continuously rewarded syllable, this could not be rationalized by either of the aforementioned two theoretical positions, but (like so many odd findings) it would fit nicely with the theory of cognitive dissonance. Festinger (1964) hypothesized that on the non-rewarded trials of a partial schedule the animal seeks "extra attractions" in the area of the goal box. Such extra attractions supposedly reduce the dissonance resulting from not obtaining an expected reward. Since, in the present experimental procedure, the syllable associated with the partial schedule is the logical candidate to become such an extra attraction, it would not be incommensurate with cognitive dissonance theory to find that the syllable became more pleasant than one associated with continuous reward.

The purpose of this experiment was to provide some information about which of the aforementioned three theoretical positions is most tenable in explaining the effects on conditioned reward value of partial schedules.

\section{METHOD}

\section{Subjects}

Ss were 20 male and 20 female undergraduates in introductory psychology courses at Vanderbilt University, who participated in the research to fulfill a course requirement.

\section{Apparatus}

Stimuli. The stimuli were four nonsense syllables: $Z O J, M Y V, K Y F$, and TEJ. Each was determined by Archer (1960) to have a similar low level of meaningfulness. In order to control for any inherent preferences, the stimuli were counterbalanced in their roles as continuously (100\%) rewarded (CR), partially $(50 \%)$ rewarded (PR), neutral $(\mathrm{N})$, and reward-loss (L) stimuli; but they remained constant in their roles for each $S$. The reward-loss syllable was one for which $S$ was required to relinquish previously acquired rewards.

Learning task. A discrimination apparatus was employed in the learning task. It consisted of two cups mounted on a board. Four lids, each bearing a card with one of the syllables on it, were placed two at a time over the cups to result in a binary choice situation on each trial. The apparatus has been described in detail by Faw \& Nunnally (1967).

\section{Learning Procedure}

Each $\mathbf{S}$ was seen individually and told that he would be able to earn some money if he could solve the problem in the experiment. $S$ was shown the lids with the syllables and told that on the bottom of each lid would be one of three tickets: "Earn 5\$," blank, or "Lose 5\$." $\mathrm{S}$ was told that two lids would be presented at a time and that he was to choose the one from which he expected a nickel and follow the instructions on the ticket. He was told that the blank ticket meant that he would neither win nor lose money on that trial. $S$ was not allowed to see which tickets were associated with which lids before the task began, nor was he informed that one of the syllables was to be partially rewarded. All possible left-right pairs of the four syllables were presented. The pairs were randomized in blocks of 12 trials, with the restriction that the same pair (i.e., with left-right position reversed) could not appear twice in immediate succession.

$S$ proceeded to make choices until he had achieved a criterion of 20 correct consecutive choices or had reached 60 trials. A correct choice was defined as choosing the lid which would maximize his earnings (e.g., CR over PR, PR over $\mathrm{N}$, and $\mathrm{N}$ over $\mathrm{L}$ ). The $\mathrm{PR}$ was associated with the "Earn $5 t$ " ticket a random $50 \%$ of the time it was presented and the blank ticket $50 \%$ of the time. After each choice $S$ was allowed to see the ticket under the alternate lid.

\section{Dependent Measures}

Reward expectancy. A "treasure hunt" method was used to observe S's expectations of reward associated with the syllables. S chose among three small boxes the one from which he expected further reward. The boxes were identical except for the syllable printed on the top side. S's CR, PR, and N were each represented on one box. $S$ was shown the three boxes with the syllables on them and told that one of them had a blackened inside, which meant he would earn a quarter. He was asked to choose the box which he thought would reward him. After making the first choice, he was asked to show his preference between the remaining two boxes. In this way an ordering of the three syllables was obtained. $S$ was not allowed to look in the boxes and was not told if he had won the quarter until the end of the session.

Semantic Differential A 15-item Semantic Differential was employed in order to obtain S's ratings of the four syllables. The 15 items represented four factors as follows: Evaluation: helpful-harmful; moral-immoral, kind-cruel, useful-useless, generous-stingy, pleasant-unpleasant, good-bad; Activity: activepassive, fast-slow; Potency: strong-weak, rugged-delicate; Understandability: familiar-strange, understandable-mysterious, clear-confusing, predictable-unpredictable. For each syllable, the order of the items was randomized. The scales were administered on paper and included eight response intervals for each item. The first eight of the items were presented with the adjectives in the order shown above (e.g., helpful-harmful), while the last seven of the items were presented with the adjectives in reversed order (e.g., harmful-helpful). $S$ was instructed to indicate his description of the syllables by relating them to the pairs of adjectives.

Locus of Control Scale

$S$ was administered an abbreviated form of McConnell's (1960) Student Opinion Survey (Part II). This scale was designed to measure Locus of Control (LC), a person's predisposition to believe that he has some control over events that befall him. Thirty of the 52 item scale were LC items, the remaining 22 
Table 1

Summary of Mean Semantic Differential Ratings of the Syllables

\begin{tabular}{|c|c|c|c|c|}
\hline & $100 \%$ & $50 \%$ & Neutral & Reward-Loss \\
\hline Scale & $(\mathrm{CR})$ & (PR) & $(\mathrm{N})$ & (L) \\
\hline Evaluation & 6.80 & 5.75 & 4.75 & 3.04 \\
\hline Activity & 6.58 & 5.28 & 3.88 & 4.77 \\
\hline Potency & 6.48 & 5.28 & 4.18 & 5.66 \\
\hline Understandability & 6.74 & 4.94 & 5.44 & 5.72 \\
\hline
\end{tabular}

being unscored buffer items.

After $S$ completed this task, he was given the money he had earned in the learning task. Each $S$ was told that he had won in the expectancy task and was given an additional quarter.

\section{Reward Expectancy}

\section{RESULTS}

Scores of 3,2 , or 1 were assigned to the CR, PR, and $\mathrm{N}$ in terms of the order of choices. The syllable chosen first received a 3, second a 2, and last a 1 . The mean scores for these three syllables across Ss and the standard deviations follow (mean is reported first): $\quad C R=2.90, .37 ; \quad P R=1.95, .31 ;$ and $N=1.15, .48$. A repeated measures analysis of variance revealed: $F(2,78)=128.74$, $\mathrm{p}<.001$. Newman-Keuls comparisons among the syllables revealed that the $C R$ was selected by $S s$ as indicating future reward significantly more often than the PR and $N(p<.01)$. Furthermore, the PR was selected significantly more often than the $\mathrm{N}$ $(p<.01)$. Only five of the $40 \mathrm{Ss}$ failed to choose the syllables in the predicted order (CR, PR, N).

\section{Semantic Differential}

The positions on the various scales in which $S$ placed each syllable were assigned numerical values from 1 through 8 ( 8 indicating a high rating in Evaluation or Activity, etc.). The mean ratings for the syllables across the items of each scale are displayed in Table 1. The standard deviations of the syllables are reported in Table 2.

Evaluation scale. The mean ratings of the syllables were submitted to an analysis of variance: $F(3,117)=35.04, p<.001$. Newman-Keuls comparisons indicated that the $C R$ was rated as having the highest evaluation $(p<.01)$. The $P R$ was given a significantly higher evaluation than the $\mathrm{N}$ and $\mathrm{L}(\mathrm{p}<.01)$. The remaining comparisons were significant at the .05 level.

Activity scale. An analysis of variance yielded: $F(3.117)=12.94, p<.01$. Newman-Keuls comparisons revealed that the $C R$ was rated significantly higher in activity than the other three syllables $(\mathrm{p}<.01$ for each comparison). The $\mathrm{N}$ was rated significantly lower in activity than the $P R(p<.01)$ and the $\mathrm{L}(\mathrm{p}<.05)$.

Potency scale. An analysis of variance yielded: $F(3.117)=15.28, p<.001$. Newman-Keuls comparisons revealed that the $C R$ was perceived as significantly more potent than the PR $(p<.01)$, the $N(p<.01)$, and the $L(p<.05)$. The $N$ was perceived as the least potent of the syllables $(p<.01$ for each comparison).

Understandability scale. An analysis of variance revealed: $F(3,117)=8.49, p<.001$. Newman-Keuls comparisons showed that the $C R$ was rated more understandable than the $P R(p<.01)$, the $N(p<.01)$, and the $L(p<.05)$.

Locus of Control Scale and Correlations

Each $\mathrm{S}$ was given a score ranging from 0 through 30 on the basis of the number of internal LC items with which he agreed. The data from this scale were employed in a correlational study including: (a) performance on the learning task (as indicated by

Table 2

Summary of Standard Deviations of Semantic Differential Ratings of the Syllables

\begin{tabular}{|c|c|c|c|c|}
\hline & $100 \%$ & $\underline{50 \%}$ & Neutral & Reward-Loss \\
\hline Scale & (CR) & $(\mathrm{PR})$ & $(N)$ & (L) \\
\hline Evaluation & 1.75 & 1.38 & 1.20 & 1.79 \\
\hline Activity & 1.93 & 1.73 & 1.78 & 2.20 \\
\hline Potency & 1.32 & 1.27 & 1.60 & 1.96 \\
\hline Understandability & 1.90 & 1.89 & 1.74 & 2.30 \\
\hline
\end{tabular}

the number of errors on the PR and (b) performance on the dependent measures. Pearson's product-moment correlation coefficient was employed in each case.

A correlation of -.36 was found between the LC scores and the number of errors made on the discrimination task when the PR was presented ( $p<.05$ for one-tailed prediction).

A coefficient of +.33 was obtained when LC scores were correlated with the mean understandability rating across all syllables $(\mathrm{p}<.05)$.

A non-significant correlation of -.23 was obtained between number of trials to criterion and the mean understandability rating across all the syllables.

\section{DISCUSSION}

The results of this experiment offer considerable support for three major conclusions: As compared with a neutral object associated with reward $100 \%$ of the time, an object associated with reward $50 \%$ of the time (a) is seen as somewhat less pleasant, but still pleasant in an absolute sense, (b) has a lower expectancy as a cue to future reward, but elicits a positive expectancy in the absolute sense, and (c) is seen as relatively mysterious or difficult to understand.

Findings (a) and (b) are consistent with predictions that follow from the literature on classical conditioning. None of the results hint of strong influences of either frustration or cognitive dissonance.

Finding (c) above suggests that partial reward engenders a type of curiosity motivation in secondarily rewarding objects. If this is so, then an important dimension of motivation has been overlooked in all studies concerning effects of reward schedules on conditioned rewards. For example, rats may turn toward a supposed secondarily rewarding stimulus in a T-maze not because they "like" it but because they are puzzled about it.

The major criticism which might be levelled against this study is that the results could be explained by the demand characteristics imposed on the Ss. College Ss may have merely acted out implicit theories concerning situations involving partial rewards. Even if that were the case, if nothing else, this study found that college students' implicit theories are much more similar to an analogy of classical conditioning than to either frustration or cognitive dissonance theories. However, an explanation of these results entirely in terms of demand characteristics is not highly tenable in the light of strikingly similar findings with children of various ages, employing somewhat different procedures, several schedules of reward, and largely different dependent measures (Duchnowski et al, 1968).

\section{REFERENCES}

AMSEL, A. Frustrative nonreward in partial reinforcement and discrimination learning: Some recent history and a theoretical extension. Psychological Review, 1962, 69, 306-328.

ARCHER, E. J. Re-evaluation of the meaningfulness of all possible CVC trigrams. Psychological Monographs, 1960, 74, No. 10.

DUCHNOWSKI, A. J., NUNNALLY, J. C., \& FAW, T. T. Conditioned reward value in discrimination learning: Methodological developments and effects of reward magnitude and reward schedules. Privately circulated research paper, Vanderbilt University, 1968.

FESTINGER, L. Conflict, decision, and dissonance. Stanford: Stanford University Press, 1964.

LONGSTRETH, L. E. Frustration and secondary reinforcement concepts as applied to human instrumental conditioning and extinction. Psychological Monographs, 1966, 80, No. 11.

McCONNELL, T. R. Locus of control as a factor in attendant selection. Unpublished manuscript, 1960.

NUNNALLY, J. C., DUCHNOWSKI, A. J., \& PARKER, R. K. Association of neutral objects with rewards: Effect on verbal evaluation, reward expectancy, and selective attention. Journal of Personality \& Social Psychology, 1965, 1, 270-274.

NUNNALLY, J. C., \& FAW, T. T. The acquisition of conditioned reward value in discrimination learning. Child Development, 1968, 39, 159-166.

NUNNALLY, J. C., STEVENS, D. A., \& HALL, G. F. Association of neutral objects with rewards: Effect on verbal evaluation and eye movements. Journal of Experimental Child Psychology, 1965, 2, 44-57.

PARKER, R. K., \& NUNNALLY, J. C. Association of neutral objects with rewards: Effect of reward schedules on reward expectancy, verbal evaluation, and selective attention. Journal of Experimental Child Psychology, 1966, 3, 324-331.

NOTE

1. While this research was in progress, the first author was receiving stipend support from PHS Training Grant No. MH08528 from the National Institute of Mental Health to the Department of Psychology. The study also was supported, in part, by a research grant from the National Institute of Child Health and Human Development, No. HD03083, to the second author. 